\section{Dental uniforms just for you}

Pastelli has been producing high quality uniforms for the dental profession since 1929. One hundred percent made in Italy, the exclusive collections offer garments that are both stylish and comfortable, making them an ideal choice for a long day in surgery.

Available from RPA Dental, the various ranges cater for all of your individual needs and preferences, right down to cut, colour and size.

There are also a number of specialist fabrics to choose from. These include:

- Lino - second only to silk for quality and beauty

- Jersey - an extremely comfortable woven cotton fabric, which offers freedom of movement

- Polycotton - a cotton and polyester mix that is both strong and easy to maintain

- Pet wash and wear - a non-iron breathable and ecological material.

With many more textiles available to pick from, you are sure to find what you are looking for with Pastelli.

To find out more about
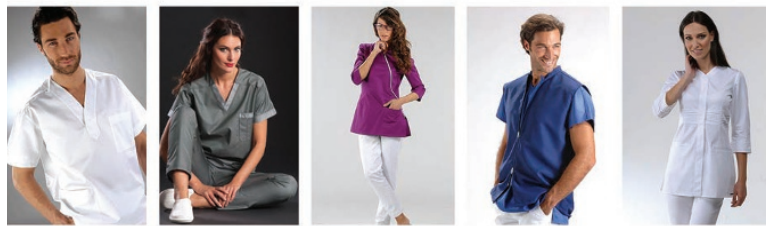

the different collections and styles available, contact RPA Dental today.

To see how RPA Dental can transform your dental practice, call 08000933 975, visit the website www.dental-equipment.co.uk or email jo@rpadental.net.

\section{Dentist launches his own toothpaste}

Dentist Dr Richard Marques of Wimpole Street Dental has launched his own dental line and the first product in the range, the Super Strong Tooth Whitening paste, is now available. The Super Strong Tooth Whitening paste is produced and developed exclusively in the USA in partnership with Dr Marques, using 100\% natural ingredients including calcium carbonate and silica for whiter teeth, with natural peppermint oil for fresh breath.

The Super Strong Tooth Whitening Paste is also available in strawberry flavour. To purchase the toothpaste, visit https:// richardjmarques.com/collections/frontpage/products/ super-strong-100-natural-tooth-whitening-paste.

\section{Handpiece-deployed caries detection system}

Lares Research has introduced dentistry's first handpiece-deployed caries detection system, Fluoresce HD. Fluoresce HD emits $405 \mathrm{~nm}$ light from a turbine or lowspeed handpiece, causing caries to fluoresce orange/ red and light green for a healthy tooth. The dentist then removes only orange/red caries, minimising removal of healthy tooth. Studies have shown fluorescence-aided caries excavation (FACE) is more accurate at detecting remaining decay than probing or caries indicator dyes, minimising the risk of reinfection and saving chair time.

Fluoresce HD can be deployed through the company's ProStyle SF and Legacy 5 turbines, any competitor's MultiFLEX ${ }^{\star}$ compatible fibre-optic turbine, and a Lares LED lowspeed motor and 1:1 fibre-optic contra angle.

For more information email cgodoy@laresdental.com or visit www.laresdental.com.

*MultiFLEX is a trademark of Kavo Dental GMBH.

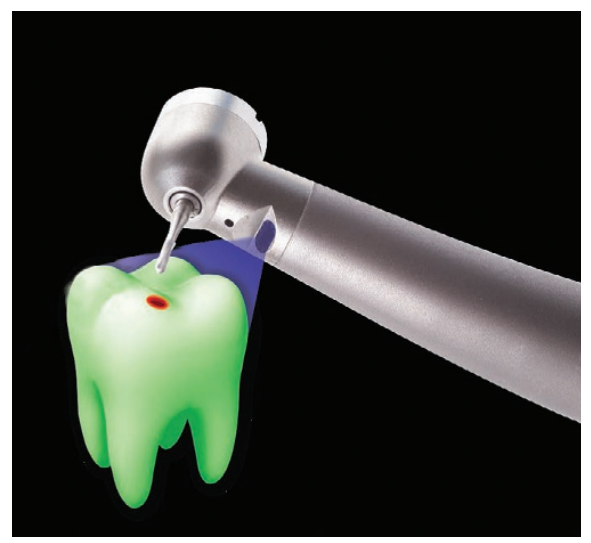

\title{
People, product and price
}

Dental Sky concentrate on what they like to call 'the 3 Ps' - people, product and price. If they employ the right people, ensure the right products are stocked, at the right price, then ordering sundries will be a breeze. It's a simple formula that's worked for them for the last 15 years and is why their business continues to grow.

An alternative mantra is to focus on three other 'Ps' ... being personal, passionate and prompt. Sounds logical, but how many times have you spoken to someone on the phone to reflect afterwards that the individual was definitely deficient in charisma and failed to deliver on their promises? Dental Sky is an independent dealer, whose staff genuinely care about their customers.

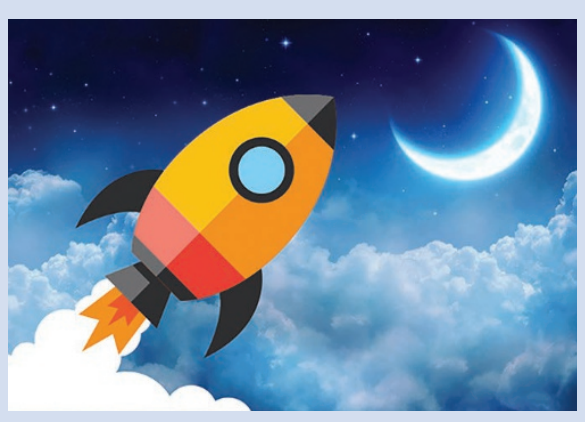

The company does not employ reps, as their salaries would add to the cost of your sundries. Better to let customers make purchasing decisions in their own time, knowing that Dental Sky will never knowingly be undersold. Price is key, and clarity a given; their offers are intentionally kept simple so you know exactly what you're getting and at what price.

If you don't have a Dental Sky account you can open one today; no credit checks, no paperwork and no hassle! Just pop online to register and you're ready to go. Visit www.dentalsky.com. 